\title{
A eficiência de alguns municípios de Mato Grosso em relação à educação por meio da Análise Envoltória de Dados
}

\author{
Mauro V. da Silva ${ }^{1}$ \\ UNEMAT, Cáceres, MT \\ Marcia C. Dal Toé2 \\ UNEMAT, Cáceres, MT \\ Tatiana R. V. da Silva ${ }^{3}$ \\ IFMT, Pontes e Lacerda, MT \\ Franciele N. dos Santos ${ }^{4}$ \\ SEDUC, Pontes e Lacerda, MT \\ Emivan F. da Silva ${ }^{5}$ \\ UNEMAT, Sinop, MT
}

\begin{abstract}
Resumo. Neste artigo utilizando-se uma formulação não paramétrica que modela a eficiência como um problema de programação linear é apresentada uma análise da eficiência de alguns municípios mato grossenses em relação à educação, ou seja, uma avaliação do desempenho desses municípios considerando os recursos disponíveis para a oferta da educação básica. Nesta análise será considerada também a comparação entre os municípios Benchmarking, com o intuito de verificar quais "eficientes"servem de modelos para os "ineficientes", além das metas e projeções. Para tanto, os resultados foram obtidos usando o modelo de Análise Envoltória de Dados, voltado aos outputs, BCC dual com folga. O modelo foi implementado usando a linguagem de modelagem algébrica AMPL e solucionado usando o solver comercial CPLEX. Os resultados obtidos são satisfatórios e possibilitam uma análise sobre os quantitativos despendidos pelos municípios para a manutenção das escolas e o retorno que se obtém, possibilitando orientação quanto a tomadas de decisões futuras.
\end{abstract}

Palavras-chave. Análise Envoltória de Dados, Análise da eficiência, Educação.

\section{Introdução}

A educação, analisada como instrumento de formação de capital humano, tem sido apontada como a resposta para o desenvolvimento econômico e social de um país, visto que, melhorias na capacitação escolar individual geram benefícios tanto individuais quanto sociais [6]. Nesse sentido, é notória a crescente preocupação em relação aos investimentos e a qualidade do setor educacional.

As escolas constituem um dos principais custos no sistema público de educação no mundo [12]. No Brasil a situação não é diferente, existe uma pressão constante para melhorar a eficiência e a qualidade do ensino público. Questões como limites de financiamento, bem como detalhes de regulação e supervisão dos gastos, são características do setor público de educação. No entanto, avaliar a eficiência do sistema educacional não é uma tarefa trivial, entre as ferramentas existentes

\footnotetext{
${ }^{1}$ mauroviegas@unemat.br.

2 marciadaltoe@unemat.br.

3 tatiana.silva@plc.ifmt.edu.br

4 franciele.santos@edu.mt.gov.br.

${ }^{5}$ emivan@unemat.br.
} 
para a análise da eficiência no âmbito educacional, a Data Envelopment Analysis (DEA) vem ganhando espaço [11].

A DEA ocupa um lugar no estudo de comparação da eficiência no setor público ao redor do mundo [12] e [14]. Existem vantagens de utilizar a DEA, por exemplo no ensino superior, pois possibilita avaliar as eficiências das universidades de diversos pontos de vista. Quando se aumenta o número de critérios de avaliações, mais universidades são consideradas eficientes [1]. Nesse sentido, a DEA permite identificar as áreas que requerem melhorias, bem como, descreve as possibilidades de desenvolvimento nessa área. Além do que já foi exposto, a DEA também permite apontar pontos fortes e fracos das unidades educacionais avaliadas, permitindo a alocação correta de recursos entre as unidades e seu tamanho ideal.

O princípio por trás da DEA consiste em determinar a melhor fronteira para as Decision Making Units (DMUs) onde se envelopa todas as DMUs ineficientes. Para ter um valor eficiente se atribui para cada DMU um valor que é a medida da sua distância até a fronteira. A utilização da DEA tem duas grandes vantagens: 1. nenhuma função de produção precisa ser especificada; 2. a possibilidade de considerar várias entradas (inputs) e saídas (outputs) ao mesmo tempo.

No que se refere ao setor educacional, de acordo com [15], emergiu na literatura um consenso sobre as entradas e saídas a serem consideradas nos estudos que visam avaliar a eficiência em educação, sendo que para as entradas foram subdivididos três grupos: (i) as características que refletem os alunos (nível de conhecimento através do aprendizado até então e socioeconômicas), (ii) as características refletindo a escola (como número de pessoal docente e não docente, despesa por aluno, tamanho da escola ou tamanho da turma) e (iii) as características que refletem os professores (como salário, experiência ou formação). Já para as saídas, o consenso é que estas devem relacionarse com os resultados de testes padronizados ou ainda com outros resultados relevantes, como por exemplo, a taxa de aprovação de alunos.

O primeiro modelo da DEA foi proposto por [2]. Desde então tem havido um crescimento no desenvolvimento e aplicações da DEA. Segundo [9] a DEA provou sua utilidade na estimativa de eficiência em cinco principais ramos de aplicações que são: bancário, saúde, agricultura, transporte e educação. Neste artigo, o interesse está em medir a eficiência em educação, com foco nas escolas públicas municipais do estado de Mato Grosso. Diversos trabalhos utilizam a DEA como ferramenta para a medição da eficiência no setor educacional tais como: [3], [5], [11], [12], [16].

As eficiências na DEA podem ser analizadas sob duas perspectivas: qualitativas ou quantitativas. De acordo com [13], na perspectiva quantitativa são consideradas variáveis de volume ou quantidade, enquanto que na qualitativa as variáveis representam desempenho ou qualidade. Nesse trabalho utiliza-se como entrada o PIB per capta, as despesas, o número de escolas, o número de professores e a extensão terrritorial. E como saída, o número de alunos atendidos, e as notas do quinto e nono anos no IDEB (Indice de Desenvolvimento da Educação Básica). Assim, essa análise é qualitativa e quantitativa, [13].

Este artigo tem por objetivo responder às seguintes questões: Dentre as cidades selecionadas para esta análise, quais são eficientes em relação à educação? Quais são ineficientes? Existe alguma relação entre o PIB per capita e a eficiência-ineficiência das cidades analisadas? Em um estado continental como Mato Grosso a extensão territorial das cidades influencia na eficiência? Os índices do IDEB poderiam ser melhorados conhecendo as ineficiências das cidades?

Este trabalho utiliza como ferramenta a DEA, que é um método não paramétrico utilizado para medir a eficiência das DMUs. Desta forma, utilizando-se os sistemas de dados públicos disponíveis, o modelo formulado é apresentado e os resultados dos testes obtidos através da implementação computacional deste modelo no solver AMPL/CPLEX são discutidos [4], [7].

Este artigo está dividido da seguinte forma: em 2. é apresentado o modelo teórico. Em 3. os principais resultados obtidos e em seguida a conclusão e perspectivas de trabalhos futuros. 


\section{Modelo DEA, BCC output dual com folga}

Nesse modelo, dado por [10], analisa-se a DMU $t$ por meio da comparação do seu desempenho com o desempenho de outras DMUs. Assim, para cada DMU o modelo deve ser executado uma vez. No caso desse artigo as DMUs são municípios do Estado de Mato Grosso. Além da eficiência dos gastos dos municípios com educação, este modelo fornece metas e projeções para as variáveis através dos Benchmarking, que consistem nas DMUs consideradas eficientes e que servem de referência para as ineficientes.

$$
\begin{aligned}
& \max E f f_{0}=\theta-\epsilon\left(\sum_{i=1}^{m} r_{i}+\sum_{j=1}^{n} w_{j}\right) \\
& \text { s.a. } \\
& x_{j t}-\sum_{k=1}^{s} \lambda_{k} x_{j k}-w_{j}=0, \quad j=1,2, \ldots, m, \quad t=1,2, \ldots, s \\
& \sum_{k=1}^{s} \lambda_{k} y_{i k}-r_{i}=\theta y_{j t}, \quad j=1,2, \ldots, n, \quad t=1,2, \ldots, s \\
& \sum_{k=1}^{s} \lambda_{k}=1 \\
& 0 \leq \theta \leq 1 \quad \mathrm{e} \quad \epsilon \geq 0 \\
& \lambda_{k} \geq 0 \quad k=1,2, \ldots, z \\
& r_{i} \geq 0, w_{j} \geq 0, \quad i=1,2, \ldots, m, \quad j=1,2, \ldots, n
\end{aligned}
$$

- $\theta$ - é uma variável, função objetivo e eficiencia da $D M U_{t}$;

- $\lambda_{k}$ - contribuições das DMUs para a eficiencia da $D M U_{t}$;

- $y_{i k} \quad$ e $x_{j k}$ - outputs $i$ e inputs $j$ da $D M U_{K}$;

- $y_{i t}$ e $x_{j t}$ - outputs $i$ e inputs $j$ da $D M U_{t}$;

- $r_{i}$ e $w_{j}$ - são as variáveis de folga da $D M U_{0}$;

\subsection{Comentários sobre o modelo matemático}

O valor de $\epsilon$ é importante na determinação das eficiências e quanto menor o valor atribuído a ele mais consistente é o resultado. Considera-se como consistente aquele resultado em que se a DMU é eficiente otimista (Eficiência Clássica) então não pode ser eficiente pessimista (Eficiência Inversa). Caso aconteça de uma DMU ser eficiente nas duas categorias, então a eficiência é falsa. Neste trabalho, a eficiência para estes casos foi avaliada através da análise dos valores não nulos das variáveis de folga. Nestes testes, usou-se três valores para $\epsilon$, a saber: 0,0001, 0,0000001 e 0,0000000001 sendo que o último forneceu resultado mais condizente com a ideia discutida aqui.

Para o cálculo das metas foram usadas as fórmulas $x_{j t}-w_{j}, j=1,2, \cdots, n, z y_{i t}+r_{i}, i=$ $1,2, \cdots, m$ e para o cálculo das projeções na fronteira as fórmulas $z y_{i t}, i=1,2, \cdots, m$. Para o cálculo das eficiências compostas, considerou-se três fórmulas $(E C-E I+1) / 2,(E C-E I+100) / 2$ e $\alpha * E C+(1-\alpha) *(1-E I)$ onde $0 \leq \alpha \leq 1, E C$ é Eficiência Clássica, $E I$ é a Eficiência Invertida. Optou-se por considerar apenas a última dessas equações, que fornecerá uma classificação mais flexível e que contempla a primeira fórmula quando $\alpha=0,5$. Nesta última fórmula, quanto maior for o valor de $\alpha$, mais benevolente será a avaliação para a DMU e, para valores baixos de $\alpha$ a avaliação será mais agressiva. 


\section{Resultados}

O modelo (1) proposto foi implementado na linguagem de modelagem algébrica AMPL por [4] e resolvido usando o solver comercial CPLEX em [8], (chamado com suas opções padrões). Foram considerados 23 municípios do estado de Mato Grosso, cuja escolha foi feita considerandose sete macro regiões e dentre os municípios de cada macro região, foram escolhidos aqueles que apresentavam todos os dados necessários, sendo: Aripuanã, Juína e Porto dos Gauchos na região Noroeste; Alta Floresta, Matupá e Peixoto de Azevedo na região Norte; Campinálolis, Ribeirão Cascalheira e Serra Nova Dourada na região Nordeste; Cláudia, Lucas do Rio Verde e Sinop na região Médio Norte; Campo Novo do Parecis, Pontes e Lacerda, São José dos Quatro Marcos na região Oeste; Cáceres, Cuiabá, Tangará da Serra e Varzea Grande na região Centro Sul; e Barra do Garças, Campo Verde, Primavera do Leste e Rondonópolis na região Sudeste.

Em relação aos dados, foram usados como insumos o PIB per capta, as despesas, o número de escolas, número de professores e extensão territorial e como produtos o número de alunos atendidos e as notas do IDEB dos alunos de quinto e nono anos, veja tabelas 1 e 2 .

Tabela 1: Dados usados para os testes (inputs)

\begin{tabular}{l|r|r|r|r|r}
\hline \multirow{2}{*}{ Cidades } & \multicolumn{5}{|c}{ Insumos } \\
\cline { 2 - 7 } & PIB percapta & Despesas & Escolas & Professores & Extensão \\
\hline Cáceres & 19896.86 & 45616907.65 & 31 & 274 & 24593.123 \\
\hline Lucas do Rio Verde & 60473.87 & 34644352.15 & 10 & 254 & 3675.221 \\
\hline Sinop & 41408.12 & 43241625.70 & 16 & 328 & 3941.958 \\
\hline \multicolumn{6}{c}{ Fonte: O próprio autor }
\end{tabular}

Neste artigo faremos a análise de eficiência apenas das cidades de Cáceres, Lucas do Rio Verde e Sinop, já que a pesquisa se encontra na fase inicial e, além disso, se trata de cidades de porte médio e com características econômicas e sociais distintas, especialmente em relação ao PIB e suas extensões territoriais. Desta forma, buscaremos aprofundar a análise dos dados e incluir mais cidades à medida em que a pesquisa for avançando, sendo este um resultado parcial que servirá como um ensaio para resultados futuros.

Tabela 2: Dados usados para os testes (Outputs)

\begin{tabular}{l|r|r|r}
\hline \multirow{2}{*}{ Cidades } & \multicolumn{3}{|c}{ Produtos } \\
\cline { 2 - 4 } & Alunos & IDEB 5 ano & IDEB 9 ano \\
\hline Cáceres & 5725 & 5.0 & 4.4 \\
\hline Lucas do Rio Verde & 5909 & 6.7 & 5.8 \\
\hline Sinop & 8815 & 5.9 & 7.0 \\
\hline \multicolumn{2}{|c}{ Fonte: O próprio autor }
\end{tabular}

Com a configuração dos parâmetros conforme apresentado acima, as cidades de Lucas do Rio Verde e Sinop apresentam eficiência clássica iguais a 1,0 e ineficiência inversa $(0,886796)$ e (0.735268), respectivamente, conforme a Tabela 3. Já a cidade de Cáceres apresenta ineficiência clássica $(0,932008)$ e eficiência inversa igual a 1,0. Assim, entende-se que os uso dos insumos pela cidade de Cáceres não está sendo feito de maneira adequada. 
Tabela 3: Resultados com o modelo BCC output dual com folga

\begin{tabular}{l|c|c|c}
\hline \multirow{2}{*}{ Cidades } & \multicolumn{3}{|c}{ Eficiência } \\
\cline { 2 - 4 } & Clássica & Inversa & Composta \\
\hline Cáceres & 0.932008 & 1.000000 & 0.466004 \\
Lucas do Rio Verde & 1.000000 & 0.887024 & 0.733893 \\
Sinop & 1.000000 & 0.735300 & 0.779410 \\
\hline \multicolumn{3}{|c}{ Fonte: O próprio autor }
\end{tabular}

Em relação às metas (Tabela 4) é preciso aumentar o número de alunos atendidos de 5.725 para 6.143 - um aumento de 7,3\%, aumentar a nota IDEB do quinto ano de 5,0 para 5,52 - um aumento de $10,4 \%$ e a nota IDEB do nono ano de 4,4 para 4,72 - um aumento de 7,2\%. Já as projeções na fronteira são 6.143 alunos atendidos, 5,36 nota IDEB para quinto ano e 4,72 IDEB para o nono ano. Neste caso, apenas a projeção da nota IDEB do quinto ano é diferente da meta.

Tabela 4: Metas e Projeções na fronteira

\begin{tabular}{l|r|r|r|r|r|r}
\hline \multirow{2}{*}{ Cidades } & \multicolumn{3}{|c|}{ Metas } & \multicolumn{3}{c}{ Projeção na fronteira } \\
\cline { 2 - 7 } & Alunos & $5^{\circ}$ ano & $9^{\circ}$ ano & Alunos & $5^{\circ}$ ano & $9^{\circ}$ ano \\
\hline Cáceres & $6.142,65$ & 5,52 & 4,72 & $6.142,65$ & 5,36 & 4,72 \\
Lucas do Rio Verde & $5.909,00$ & 6,70 & 5,80 & 5909,00 & 6,70 & 5,80 \\
Sinop & $8.815,00$ & 5,90 & 7,00 & $8.815,00$ & 5,90 & 7,00 \\
\hline \multicolumn{6}{c|}{ Fonte: O próprio autor }
\end{tabular}

Observe na Tabela 5 os Benchmarking das três cidades analisadas, pode-se verificar que Sinop é a referência para Cáceres e que, além disso, Sinop é modelo para outras cidades como Alta Floresta, Aripuanã, Campo Novo do Parecis, Juína e Pontes e Lacerda. Enquanto Lucas do Rio Verde é referência para Barra do Garças, Campo Verde, Primavera do Leste e Rondonópolis.

Tabela 5: Resultados com o modelo BCC output dual com folga

\begin{tabular}{l|c|c|c}
\hline \multirow{2}{*}{ Cidades } & \multicolumn{3}{|c}{ Benchmarking } \\
\cline { 2 - 4 } & $\lambda_{23}$ & $\lambda_{66}$ & $\lambda_{131}$ \\
\hline Alta Floresta & 0.00 & 0.00 & 0.22 \\
Aripuanã & 0.00 & 0.00 & 0.23 \\
Barra do Garças & 0.00 & 0.07 & 0.00 \\
Cáceres & 0.00 & 0.00 & 0.07 \\
Campo N. Parecis & 0.00 & 0.00 & 0.16 \\
Campo Verde & 0.00 & 0.26 & 0.13 \\
Juína & 0.00 & 0.00 & 0.13 \\
Lucas do Rio Verde & 0.00 & 1.00 & 0.00 \\
Pontes e Lacerda & 0.00 & 0.00 & 0.10 \\
Primavera do Leste & 0.00 & 0.66 & 0.00 \\
Rondonópolis & 0.00 & 0.38 & 0.02 \\
Sinop & 0.00 & 0.00 & 1.00 \\
\hline \multicolumn{3}{|c}{ Fonte: O próprio autor }
\end{tabular}

Tendo em vista a análise apresentada, ao se considerar os insumos da cidade de Cáceres os 
indicadores apontam que é preciso atender mais alunos e melhorar as notas do IDEB das séries analisadas, como pode ser observado na Figura 1.

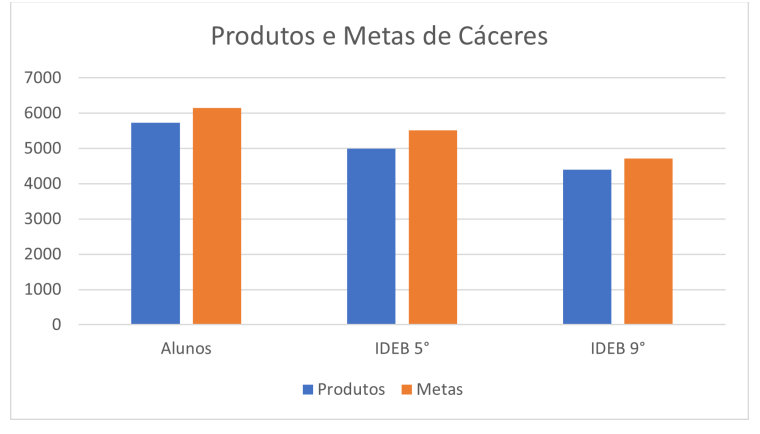

Figura 1: Produtos observados e metas para Cáceres. (notas multiplicadas por mil).

O PIB percapta de Sinop é mais que o dobro do PIB de Cáceres, e a despesa com educação por escola de Sinop na ordem de $\mathrm{R} \$ 2.702 .601,60$ é maior que o de Cáceres na ordem de R $\$ 1.471 .513,15$. Apesar dessas diferenças nos insumos Sinop é apontado como eficiente pelo modelo e Cáceres não. Isso se deve principalmete ao fato dos resultados (produtos) de Sinop serem melhores que os de Cáceres. A saber: Sinop atende 8.815 alunos e o rendimento no IDEB foi de 5, 9 para o quinto e 7.0 para o nono ano enquanto Cáceres atende 5725 alunos, o que corresponde a $53,9 \%$ do número de alunos atendidos por Sinop e o rendimento IDEB é de 5, 0 e 4, 4 no quinto e nono anos, respectivamente.

\section{Conclusões}

Tendo em vista os resultados apresentados ao considerar como insumos o PIB per capita, a despesa anual com educação, o número de escolas, o número de professores e a extensão territorial e como produtos o número de alunos e o IDEB do quinto e nono anos, dentre as cidades consideradas para análise, as eficientes foram: Sinop e Lucas do Rio Verde. Os indicadores apontam que o município de Cáceres não é eficiente em relação aos gastos públicos em educação e que é preciso aumentar o número de alunos atendidos e melhorar as notas do IDEB do quinto e nono anos do ensino fundamental. A cidade de Sinop que foi considerada referência para Cáceres possui PIB per capita maior que o dobro do PIB de Cáceres, e a despesa com educação também é maior, consequentemente Sinop atende um número maior de alunos em comparação com Cáceres e apresenta um desempenho superior em relação ao IDEB. Em relação ao município de Lucas do Rio Verde, Sinop apresenta menor PIB per capita e no entanto consegue atender um número maior de alunos e apresentar um bom resultado do IDEB.

Para trabalhos futuros será proposto uma expansão da análise a todas as cidades de Mato Grosso, bem como aumento do número de inputs e outputs.

\section{Referências}

[1] Aoki, S., Inoue, K., Gejima, R., Data envelopment analysis for evaluating Japanese universities. Artif Life Robotics 15, 165-170 (2010). https://doi.org/10.1007/s10015-010-0786-7

[2] CHARNES, A., COOPER, W.W., RHODES, E., Measuring the efficiency of decision making units. European Journal of Operational Research, n. 2, p. 429-444, 1978. 
[3] De Witte, K., López-Torres, L. Efficiency in education: A review of literature and a way forward. [S. l.: s. n.], 2017. Disponível em: https://doi.org/10.1057/jors.2015.92.

[4] Fourer, R., Gay, D. M., Kernighan, B. W. AMPL: a modeling language for mathematical programming. 2. editora Pacific Grove: Thomson/Brooks/Cole, 2003.

[5] Garabedian, F. B. P., KORN, D. Generalized Convexity and Optimization. Berlin, Heidelberg: Springer Berlin Heidelberg, 2009, ISSN 0075-8442.(Lecture Notes in Economics and Mathematical Systems).v. 616 Disponível em: https://doi.org/10.1007/978-3-540-70876-6.

[6] Goldemberg, J. O repensar da educação no Brasil. Estudos Avançados, [s. 1.], v. 7, n. 18, 1993. Disponível em: https://doi.org/10.1590/s0103-40141993000200004

[7] IBM CORP., IBM. V12. 1: User's Manual for CPLEX. International Business Machines Corporation, [s. 1.], v. 12, n. 1, p. 481, 2009. Disponível em: http://scholar.google.co.uk/scholar?hl=en\&q=cplex + manual\&btnG $=\&$ as_sdt $=1,5 \&$ as_sdtp $=$ \#4\%5Cnhttp://scholar.google.com/scholar?hl=en\&btnG=Search\&q=intitle:User's+Manual + for + CPLEX $\% 231$

[8] Ilog. CPLEX Optimization subroutine library guide and reference, version 11.0. Incline Village: ILOG, 2008.

[9] Liu, J. S., Louis, Y. Y. L., Wen-Min, L., Lins, B. J. Y.,A survey of DEA applications. omega, pg. 893 - 902, 2013

[10] Mello, J. C. C. B. S. de, Meza, L. A., Gomes, E. G., Neto L. B., Curso de análise de envoltória de dados XXXVII Simpósio Brasileiro de Pesquisa Operacional - SBPO 27 a 30/09/2005, Gramado - RS

[11] Mota, T. R. A., Meza, L. A. The use of dea as a tool to evaluate public expenditure on education: An analysis of the cities of the State of Rio de Janeiro. Anais da Academia Brasileira de Ciencias, [s. 1.], v. 92, n. 2, p. 1-13, 2020. Disponível em: https://doi.org/10.1590/00013765202020190187

[12] Nazarko, J., Saparauskas, J. Application of dea method in efficiency evaluation of public higher education institutions. Technological and Economic Development of Economy, [s. 1.], v. 20, n. 1, p. 25-44, 2014. Disponível em: https://doi.org/10.3846/20294913.2014.837116

[13] Nuintin, A. A., Eficiência da aplicação de recursos públicos nas Universidades Federais. 169 f. 2014. Tese (Doutorado) - Universidade Federal de Lavras, Programa de Pós-graduação em Administração. Lavras, 2014.

[14] Odeck, J., Evaluating Target Achievements in the Public Sector: An Application of a Rare Non-Parametric DEA and Malmquist Indices. Journal of Applied Economics, v. 8, number 1, pg. 171 - 190, 2005.

[15] Thanassoulis, E., Witte, K. de, Johnes, J., Johnes, G., Karagiannis, G., Portela, C. S. Applications of Data Envelopment Analysis. In: Zhu, J. Data envelopment analysis: a handbook of empirical studies and applications. New York : Springer, 2016. P. 367-438.

[16] Zhu, J. Data Envelopment Analysis Associate Series Editor. [S. 1.: s. n.], 2016. 